\title{
Cues for different diagnostic patterns of interpersonal violence in a psychiatric sample: an observational study
}

Dalila Talevi ${ }^{1 *}$, Alberto Collazzoni ${ }^{1}$, Alessandro Rossi ${ }^{1}$, Paolo Stratta ${ }^{2}$, Monica Mazza ${ }^{1}$, Francesca Pacitti ${ }^{1}$, Manuela Costa', Claudio Crescini ${ }^{1}$ and Rodolfo Rossi ${ }^{3}$

\begin{abstract}
Background: Interpersonal violence has increased as a health concern, especially in psychiatry practice, over the last decades. Nevertheless, most patients with stable mental disorders do not present an increased risk of violence, and mental disorder is not a necessary or sufficient cause of violent behaviours. People with mental disorders endorse more often a number of risk factors for violence that could confound this association, such as young age and male gender. The aim of this study was to investigate the effect of age, gender, and diagnosis on reported levels of interpersonal violence in a sample of people with severe mental illness.
\end{abstract}

Methods: The sample was composed of 160 inpatients: 73 with a psychosis within the schizophrenia spectrum, 53 with a mood disorder and 34 with a personality disorder. All patients enrolled in the study were assessed for experiences of victimization and perpetration of interpersonal violence using the Karolinska Interpersonal Violence Scale interview. Demographic variables were also collected.

Results: Both violence perpetration and victimization negatively correlated with age. Compared to males, females were exposed to higher degree of victimization in childhood and adulthood, whereas males were more involved in the perpetration of violence in childhood. Personality disorders were associated with higher levels of interpersonal violence, both perpetration and victimization; an interaction effect of gender and diagnosis was also observed for violence perpetration in adulthood. Distinct patterns of interpersonal violence did emerge for the diagnostic groups with mood disorder showing a victimization pattern, personality disorders a perpetration pattern and psychoses less defined patterns.

Conclusions: The main finding is that psychotic disorders, mood disorders and personality disorders have different patterns of violent experiences interacting with age and gender. This study offers a better understanding of how gender and age could affect violent behaviours. Moreover, study findings may increase the comprehension of the reason why some mental disorders, compared to others, are more associated with the risk of victimization or perpetration of violence. These patterns could have pathophysiological or pathoplastic meaning addressing clinical and diagnostic trajectories and they could interact with other intervening risk factors.

Keywords: Interpersonal violence, Mental illness, Gender, Young age, Psychosis, Mood disorders, Personality disorders

\footnotetext{
* Correspondence: dalila.talevi@gmail.com

'Department of Biotechnological and Applied Clinical Sciences (DISCAB), University of L'Aquila, L'Aquila, Italy

Full list of author information is available at the end of the article
}

(c) The Author(s). 2020 Open Access This article is licensed under a Creative Commons Attribution 4.0 International License, which permits use, sharing, adaptation, distribution and reproduction in any medium or format, as long as you give appropriate credit to the original author(s) and the source, provide a link to the Creative Commons licence, and indicate if changes were made. The images or other third party material in this article are included in the article's Creative Commons licence, unless indicated otherwise in a credit line to the material. If material is not included in the article's Creative Commons licence and your intended use is not permitted by statutory regulation or exceeds the permitted use, you will need to obtain permission directly from the copyright holder. To view a copy of this licence, visit http://creativecommons.org/licenses/by/4.0/ The Creative Commons Public Domain Dedication waiver (http://creativecommons.org/publicdomain/zero/1.0/) applies to the data made available in this article, unless otherwise stated in a credit line to the data. 


\section{Background}

Interpersonal violence (IV) has become a major public health issue over the last decades [1], being a significant cause of morbidity and mortality worldwide [2, 3]. IV has increased as a health concern especially in psychiatry practice [4]. IV encompasses two main components: expression (i.e., perpetration) of and exposure (i.e., victimization) to violence. It's important to distinguish victimization and perpetration experiences given that the two types of violence are associated with different risk factors, health consequences and management [5].

Most patients with stable mental disorders do not present an increased risk of violent behaviour [6] and they are more likely to be victims than perpetrators [79]. However, schizophrenia, mood disorders, posttraumatic stress disorder, personality disorders and substance use disorders are related to a high occurrence of violent behaviours especially when active symptoms or a relapse are present $[6,8,10-13]$. A broad body of research focused on the detection of risk factors for violence perpetration related to psychiatric morbidity. Most epidemiological and clinical studies support the notion that mental disorders provide a modest contribution to violence risk among adults $[11,14,15]$, concluding that a mental disorder is not a necessary or sufficient cause of violent behaviours [7], nor an independent predictor [16]. Patients who more frequently reported violent experiences showed other factors associated with violence, such as historical, dispositional, and contextual ones [6, $14,16]$.

Among demographic variables associated with violence, age and gender have received large attention so far $[2,11,17]$. Literature consistently reports that younger age is associated with high levels of both perpetration and victimization in community samples as well as clinical and forensic populations [2, 7, 9, 11, 12, 16-20]. The role of gender in IV is largely debated and controversial, with both evidence supporting gender imbalance in violence perpetration (i.e., males $>$ females, $[6,7,11$, 16]; females > males, [12]), as well as studies reporting similar rates in males and females [21-24]. The gender pattern of victimization is more consistent, with women being overly victimized compared to men $[8,9,12,21$, 24-28]. Globally, evidence suggests that men represent the majority of perpetrators and women the majority of victims [29].

\section{Aims and hypotheses}

Suffering from a severe mental illness (SMI) might increase the probability of experiencing violence; however, people with SMI endorse more often a number of risk factors for violence that could confound this association.

The goal of this study is to investigate how the diagnosis of a SMI interact with age and gender in the context of violent experiences. We aimed to detect any diagnosis-life stages and diagnosis-gender pattern associated with violence perpetration and victimization. The Karolinska Interpersonal Violence Scale (KIVS) was used as it measures expression of and exposure to violence, consistent with a victimization and a perpetration pattern of interpersonal violence, respectively. Our hypothesis is that different victimization/perpetration patterns exist for psychoses within the schizophrenia spectrum and other psychotic disorders (SSOPD), mood disorders and personality disorders when combining diagnosis with age and with gender.

\section{Methods}

\section{Study design}

This study had a cross-sectional observational design. Data were collected at the psychiatric unit of the L'Aquila San Salvatore Hospital, Italy, in collaboration with the Department of Biotechnological and Applied Clinical Sciences of University (DISCAB) from December 2016 to March 2018. All the procedures and the research project were approved by the local ethics committee.

\section{Participants and procedures}

Among 230 consecutively admitted patients who were eligible for the study, 164 agreed to participate $(71.3 \%$ of eligible patients); 66 (28.7\% of eligible patients) refused to participate for many reasons. Our final sample was of 160 patients (69.6\% of eligible patients) hospitalized for a SMI (i.e., mood disorders, SSOPD, and personality disorders) index episode. Four patients showed more than $5 \%$ of missing data and were therefore not involved in statistical computations.

The primary psychiatric diagnosis was established by senior psychiatrists (AR, FP), according to the Diagnostic and Statistical Manual of Mental Disorders $5^{\text {th }}$ edition [30] criteria. Participants were appropriately informed of the possibility of being recruited into the study through clinical interview and information materials posted on the bulletin boards of the ward. Each patient gave written informed consent prior to inclusion. Subjects who provided consent were interviewed for socio-demographic characteristics and evaluated for assessing violence perpetration and victimization experiences. Evaluations were performed when patients achieved remission, in order to minimize bias due to variations of clinical conditions. Exclusion criteria were: a) age $>65$ years, b) language barriers, c) impaired consciousness, d) severe aphasia, and e) intellectual disability or other cognitive deficits.

\section{Assessment tool for interpersonal violence}

Violence victimization and perpetration were assessed using the KIVS. KIVS is composed of four rating scales 
assessing exposure to violence ("victim of violence" subscales) and expressed violence behaviour ("used violence" subscales) in childhood (between 6 and 14 years of age) and adulthood (from 15 years upwards). The steps of the KIVS are defined by short statements about concrete examples of violent episodes of increasing severity and frequency that could have occurred throughout the respondent's lifetime. The ratings ( $0-5$ for each subscale, in total maximum of 20) are based on a semi-structured interview performed by trained clinicians. KIVS is specific for IV and distinguished aggressive acts from thoughts. It was validated against several questionnaires measuring aggression and acts of violence and has good psychometric properties [31]. Moreover, it allows for use of composite scores of its subscales [32]. It has been used in several suicide research studies $[19,33-36]$ and in observational studies within clinical samples [32, 37-40]. In the current study, we used the four subscales separately as well as the composite scores of lifetime (from childhood to adulthood) expressed violence and exposure to violence ("lifetime expressed violent behaviour" composite score and "lifetime exposure to violence" composite score). Upon completion of a back-translation process, the Italian version was administered after authorization by original authors [41].

\section{Statistical analysis}

Data were analyzed using the Statistical Package for Social Sciences (SPSS ${ }^{\circ}$, version 24, IBM, U.S.A.). Mean \pm $\mathrm{SD}$, and frequencies were calculated for descriptive analysis. Diagnostic differences in violence experiences were examined using a one-way analysis of variance (ANOVA) with Bonferroni's post hoc test among the three diagnostic groups.

In order to investigate gender differences on levels of violence, an independent-samples $t$-test was calculated for all the KIVS subscales. $t$-test was initially performed on the whole sample, and subsequently it was repeated stratifying the sample by diagnosis and gender.

Bivariate Pearson correlations were calculated between age and the composite KIVS scores of lifetime expressed violence and exposure to violence.

Two-way ANOVAs were conducted to test the diagnosis-gender pattern of perpetration and victimization.

Finally, to test the diagnosis-life stages pattern, bivariate Pearson correlations was calculated between the four KIVS subscales.

\section{Results}

\section{Descriptive characteristics}

The sample was composed of 86 male patients (53.80\%) and $74(46.30 \%)$ female patients, with a mean age of $41.15 \pm 12.60$ years. About $90 \%$ of the participants were of Italian nationality and almost $99 \%$ were Caucasians. Most of them belonged to the lower class, had a medium level of education (secondary or high school, 76.20\%), were single $(60.60 \%)$ and unemployed $(48.80 \%)$. Seventythree subjects were diagnosed as affected by SSOPD (45.6\%), 53 by mood disorders (33.1\%), and 34 by personality disorders (21.3\%; Table 1).

The KIVS mean scores in the total sample were: Total score $=4.91 \pm 3.57$; Used violence as a child subscale $=$ $0.48 \pm 0.84$; Used violence as an adult subscale $=1.03 \pm$ 1.28; Victim of violence in childhood subscale $=1.81 \pm$ 1.59; Victim of violence in adulthood subscale $=1.61 \pm$ 1.47.

Incidentally, even though it was not a primary aim of the study, KIVS differences between the clinical sample and a matched control group $(n=160)$ was calculated. The control group showed lower scores for all the KIVS subscales (Total score $=1.16 \pm 0.80$; Used violence as a child subscale $=0.22 \pm 0.63$; Used violence as an adult subscale $=0.12 \pm 0.50$; Victim of violence in childhood subscale $=0.44 \pm 0.32$; Victim of violence in adulthood subscale $=0.37 \pm 0.26$ ). All these differences were significant (two-tailed independent t-test at the 5\% level, $p<.001)$. The same results were confirmed in an expanded sample [41].

\section{Diagnostic, age and gender differences}

The personality disorders group had higher Used violence as an adult $[\mathrm{F}(2,157)=3,64, p=.03]$, Victim of violence in adulthood $[\mathrm{F}(2,157)=5.05, p=.01]$, and KIVS total score $[\mathrm{F}(2,157)=4,96, p=.01]$ values than the patients with mood disorders. No other significant differences were found between the groups (Table 2).

In the total sample, violence (KIVS total score) negatively correlated with age $(r=-.33)$, both victimization ("lifetime exposure to violence" composite score; $r=$

Table 1 Demographic characteristics of the sample $(N=160)$

\begin{tabular}{lll}
\hline & & Mean (SD)/n (\%) \\
\hline Age & & $41.15(12.60)$ \\
& Single & $97(60.6)$ \\
& Married/cohabitant & $31(19.4)$ \\
& Separated/divorced & $32(20)$ \\
Education level & Low education & $7(4.4)$ \\
& Medium education & $122(76.2)$ \\
& High education & $31(19.4)$ \\
Occupational status & Unemployed & $78(48.8)$ \\
& Employed & $44(27.5)$ \\
& Others & $38(23.7)$ \\
\hline
\end{tabular}

Note: Low education refers to illiteracy and primary school certificate; medium education refers to secondary and high school certificate; high education refers to graduation and post-graduate degree. Others (Occupational status) refers to odd jobs, pensioners, students and housewives 
Table 2 One-way ANOVA analysis evaluating the differences on KIVS subscales and total score in the three diagnostic groups (Mean + SD)

\begin{tabular}{llllll}
\hline Variables (KIVS scores) & $\begin{array}{l}\text { SSOPD (1) } \\
(n=73)\end{array}$ & $\begin{array}{l}\text { Mood Disorders (2) } \\
(n=53)\end{array}$ & $\begin{array}{l}\text { Personality Disorders (3) } \\
(n=34)\end{array}$ & F & Post hoc comparison $^{\mathbf{a}}$ \\
\hline Used violence as a child & $0.48(0.88)$ & $0.34(0.68)$ & $0.68(0.94)$ & 1.69 & - \\
Used violence as an adult & $1.01(1.22)$ & $0.75(1.11)$ & $1.5(1.54)$ & $3.64^{*}$ & $3>2^{*}$ \\
Victim of violence in childhood & $1.77(1.41)$ & $1.72(1.70)$ & $2.06(1.79)$ & 0.53 & - \\
Victim of violence in adulthood & $1.63(1.37)$ & $1.21(1.20)$ & $2.21(1.84)$ & $5.05^{* *}$ & $3>2^{* *}$ \\
Total score & $4.88(3.21)$ & $4(3.27)$ & $6.41(4.29)$ & $4.96^{* *}$ & $3>2^{* *}$ \\
\hline
\end{tabular}

Note. SSOPD = schizophrenia spectrum and other psychotic disorders; KIVS = Karolinska Interpersonal Violence Scale

${ }^{*} p<.05{ }^{* *} p<.01$

a Bonferroni post hoc

-.28) and perpetration ("lifetime expressed violent behaviour" composite score; $r=-.27$ ).

Used violence as a child score is higher in males $(0.64 \pm$ $0.90)$ than in females $(0.28 \pm 0.71)$, [ $(158)=2.77, p=.01$ ] Victim of violence in childhood score is higher in females $(2.08 \pm 1.74)$ than in males $(1.58 \pm 1.41)$, [ $\mathrm{t}(158)=-1.97$, $p=.05$ ] as well as Victim of violence in adulthood score (females: $1.92 \pm 1.67$; males: $1.35 \pm 1.21)$, [t $(158)=-2.43$, $p=.02]$. No significant differences were found for Used violence as an adult (females: $0.89 \pm 1.21$; males: $1.15 \pm 1.33$ ) and KIVS total score (females: $5.16 \pm 3.62$; males: $4.70 \pm$ 3.53).

\section{Diagnosis-life stages pattern}

Significant and positive correlations between the different types of violence at two life stages (i.e., childhood and adulthood) in the three diagnostic groups have been found (Fig. 1).

The Victim of violence in childhood subscale showed a significant, positive, weak correlation with the Victim of violence in adulthood subscale in SSOPD patients $(r=.24)$. Similarly, the Victim of violence in childhood and the Victim of violence in adulthood subscales showed a significant, positive, but higher, correlation in mood disorders sample $(r=.52)$. On the contrary, in the personality disorder sample, the Used violence as a child and the Used violence as an adult subscales showed a significant, positive correlation $(r=.57)$.

The Used violence as a child subscale had a significant, positive, but small correlation with the Victim of violence in childhood subscale only in the SSOPD sample $(r=.25)$. Instead, the Used violence as an adult subscale is significantly and positively correlated to the Victim of violence in adulthood subscale in SSOPD $(r=.29)$ and mood disorder $(r=.37)$ samples.

\section{Diagnosis-gender pattern}

The $t$-test, when the sample was broken down by diagnosis, showed significant gender differences for the KIVS subscales in mood and personality disorders groups, but not in SSOPD one (Fig. 2).
The two-way ANOVAs reported a statistically significant interaction between the effects of gender and diagnosis on Used violence as an adult subscale $[F(2,154)=$ 2.99, $p=.05]$. To better understand if the Used violence as an adult is different in the considered diagnoses for gender, we analyzed males and females separately. We ran a one-way ANOVA for just diagnoses on either group. The one-way ANOVA performed on Used violence as an adult subscale $[\mathrm{F}(2,83)=6.40, p=.03]$ in all of the three-diagnosis, showed that the male patients with a diagnosis of personality disorder had higher scores than the other patients. No differences were reported between the other diagnoses. Finally, no differences have been found in female group. There was not a statistically significant interaction between the effects of gender and diagnosis on Victim of violence in childhood [F $(2,154)=2.76, p=.07]$, on Victim of violence in adulthood $[\mathrm{F}(2,154),=.16, p=.85)$, on Used violence as a child subscales [F $(2,154),=1.40, p=.25]$, and on KIVS total score $[\mathrm{F}(2,154),=2.12, p=.12]$ (Fig. 2).

\section{Discussion}

\section{Main findings}

The main finding of this study is that psychotic disorders (SSOPD), mood disorders and personality disorders have different patterns of violent experiences when combined with age and gender.

Overall, in our sample, people with personality disorders showed the highest levels of both violence perpetration and victimization, reporting significant differences compared to mood disorders in adulthood. Younger age was associated with both perpetration and victimization. Females were more often victimized than males, both in childhood and adulthood, whereas males engaged more often in violent behaviours than females in early life. When we explored the episodes of violence victimization and perpetration that occurred in childhood and adulthood, a distinct diagnosis-life stages pattern did emerge for the three diagnostic groups, with mood disorders showing a strong victimization pattern, personality disorders a strong perpetration pattern and SSOPD less 

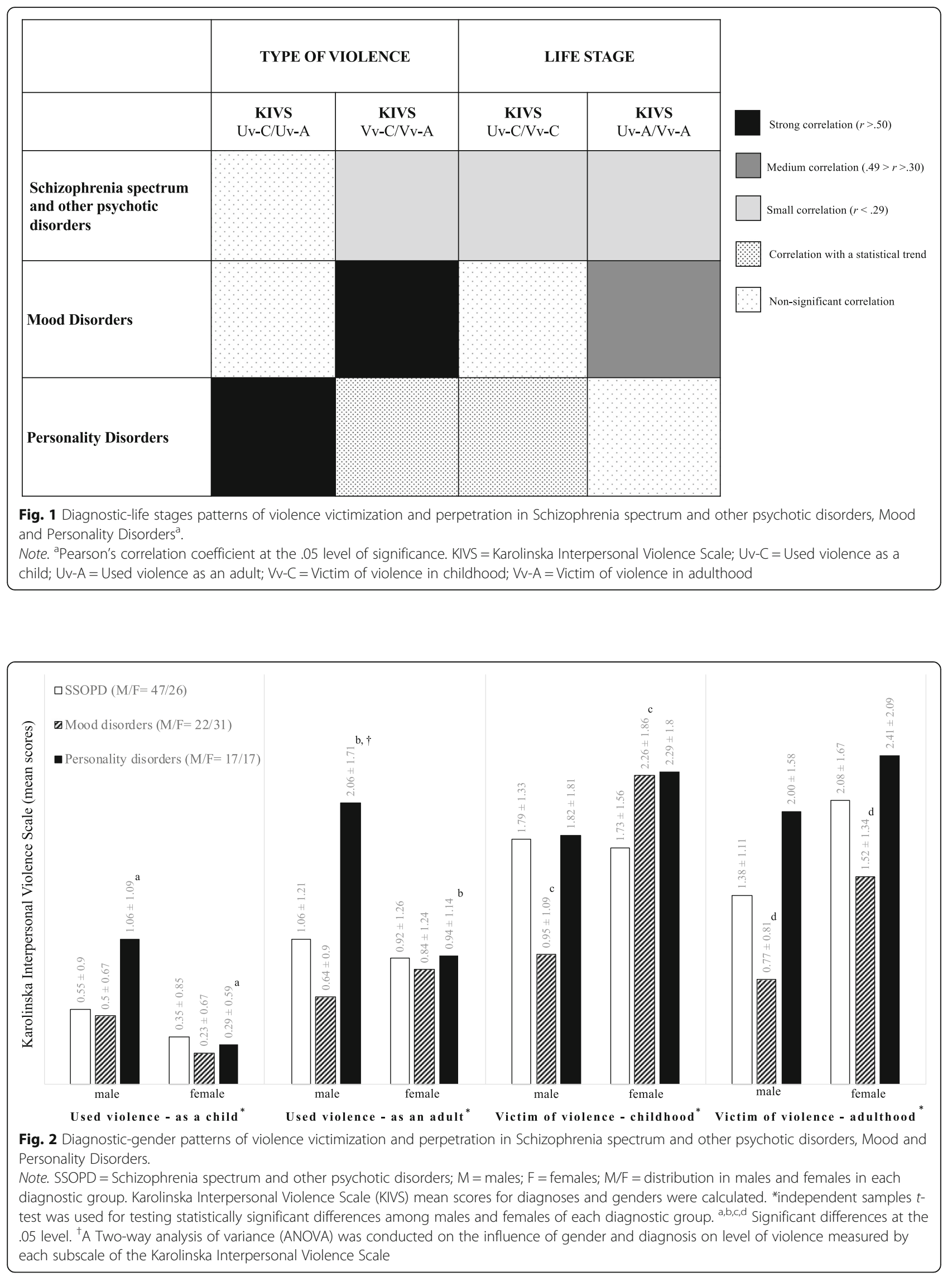
defined patterns. For people affected by a mood disorder, victimization in childhood is positively correlated with victimization in adulthood. Moreover, victimization was positively correlated with perpetration in adults with mood disorders, based on a medium "victimization-perpetration association pattern". This finding is in line with the evidence that victimized subjects are more likely to engage in violent events $[9,12]$. On the contrary, in personality disorders, engaging in violent behaviour in childhood is positively and strongly correlated with violent acts perpetrated in adulthood. People with SSOPD showed weaker "childhood-adulthood victimization association pattern" and "victimization-perpetration association pattern" (both childhood and adulthood) than other patients (see Fig. 1).

Our findings on the diagnosis-gender pattern showed that male subjects suffering from a personality disorder had higher scores than other patients in "Used violence as an adult subscale". We could claim that in these subjects a cluster of risk factors (i.e., gender, marital status, diagnosis, substance misuse) interact with each other increasing exponentially the risk of violent behaviours.

According to a more general perspective, our findings about the gender-diagnosis interaction allows some comments. Firstly, the absence of the interaction in childhood was expectable: a psychiatric condition might not have developed or be pervasive yet at that time. Secondly, gender and diagnosis had an interaction effect only on expression of violence in adulthood, that was the only subscale not to show a gender difference, although showing differences among diagnostic groups. This finding could mean that a specific SMI in a specific gender might have a more pervasive impact on the perpetration of violence, not on victimization. These findings should be interpreted carefully given their inherent methodological shortcomings.

Males had higher scores in used violence subscales than females with the same diagnosis and in the same period of life; on the contrary, females had the highest scores on victimization subscales. This pattern did not repeat for "used violence as an adult" and "victim of violence in childhood": in the first case, females with mood disorders reported greater expression of violence than males; in the second case, female victims of violence in childhood with a mood disorder got higher scores than females with SSOPD. Moreover, males with SSOPD reported higher levels of victimization in childhood then females. Although the results are not statistically significant in most cases, they and their graphic representations provide stimulating cues. For example, mood disorders showed to have a trend of a lower involvement in both violence perpetration and victimization, in particular for males; both males and females with personality disorders showed a trend of greater involvement in the expression of and in the exposure to violence, respectively (see Fig. 2).

\section{Previous literature}

Overall, our findings are consistent with pre-existing literature. As regards the relation between mental disorders and violence, a recent large American populationbased study confirms that personality disorders show higher odds for violence perpetration [11], compared to other mental disorders. Cluster B and paranoid personality disorders are considered the most likely linked to violent offending and aggression [42, 43], to suicidal behaviours and criminal arrest [44]. The association between personality disorders and violence perpetration is possibly linked to their intrinsic impulsiveness, substance abuse and bio-psychological mechanisms $[6,45]$. As regards SSOPD, only a modest relation with violent acts has been found in several large population-based studies [46]; regarding mood disorders, some evidence suggests an increased risk of engaging in violence in particular those with bipolar disorder [47].

Our results regarding the correlation between age and violence are consistent with other researches $[2,9,11$, $15,17,19]$. Using the KIVS, which distinguishes exposure to and expression of violence in childhood and adulthood, we demonstrated the strong, inverse correlation between age and both perpetration and victimization. Further studies are necessary to understand if the young are actually more violent than older people, or they merely tend to remember more often or disclose more freely violent episodes.

With regard to gender, the results concerning the victimization pattern are in line with previous literature reporting that females are the main victims of IV $[8,9$, $12,21,25-28]$. When broken down by diagnosis, the exposure to violence both in childhood and adulthood was more frequent in females for mood disorders only. This finding is somewhat in line with studies reporting an association between victimization and the presence of a mood disorder in females [8, 11, 27]. As regards perpetration of violence, we replicated that males are more likely than females to act aggressively in childhood [19, $31,35,48]$. On the other hand, we did not find a significant difference for violence perpetration in adulthood between males and females, contrary to previous studies. This finding could be due to several reasons, including the small sample size. The largest epidemiological studies on this issue found that males commit acts of violence at greater rates than females [11, 14, 15, 49]; only the National Institute of Mental Health Clinical Antipsychotic Trials of Intervention Effectiveness (CATIE), which investigated violent outcomes in schizophrenia patients as part of a large multisite randomized clinical trial, found an association between female, rather than 
male gender, and minor violence [50]. A paucity of other studies came to different conclusions: in an extensive review, Gillies \& Brien [18] reported that, due to inconsistent results from literature, no clear "gender-violence perpetration" pattern could be established. Hamberger [21] conducted a "gender analysis" about intimate partner violence, finding no difference between males and females in terms of "frequency", whereas males engage in more severe violent behaviours. Lastly, Desmarais and colleagues [9], pooling data from five studies on individuals with mental disorders from the United States, found that women reported significantly higher rates of violence perpetration than men, suggesting that this finding might reflect, for women in the community, increased opportunity of being violent or the more likelihood to disclose violence-related experiences.

Taken together, these findings suggest that people with personality disorders are at greater risk for perpetuating cycles of violence perpetration, whereas people with mood disorders are more predisposed to be a victim across the life span. In another perspective, we could argue that being a victim of violence in early life plays a role in developing a mood disorder and predisposes to revictimization. Regards to SSOPD, results are more ambiguous, so no comment can be made regarding any specific pathway or pattern of violence.

Although somehow in line with previous literature $[8$, $10,11,42,45]$ future research comparing diagnoses, gender and age impact, including larger size samples and using more sophisticated methodological approaches are warranted.

\section{Limitations}

This study presents a number of limitations. The crosssectional nature of the study design is a major limitation making impossible to confirm any causal association between the variables of interest. As a matter of fact, we could not sustain that suffering from a SMI represents a condition favoring experiences of violence.

A second limitation is the relatively small sample size. Furthermore, most of participants belonged to the same ethnicity and socioeconomic class, so the chance of detecting any effects due to interethnic or social differences played a meaningful role was limited.

Thirdly, the study solely based on KIVS, a clinical interview, whose ratings was not compared with other measurements, such as self-report questionnaires. Higher rates in self-reported victimization and perpetration of violence have been shown in comparison to clinical interview [31], so, in this study, the participants' ratings could depend on their openness to disclose their experiences to others, not on the truth of the facts.

Moreover, we did not perform an interrater reliability analysis of this clinician-administered interview, so we cannot exclude inhomogeneity in the ratings given by various clinicians. Finally, previous researches based on KIVS failed to consider issues of ethnic diversity since the instrument was administered to Sweden and Italian populations only; therefore, the findings reported cannot be generalized. Even if gender distribution across diagnoses reflects that reported in the literature, this finding could affect results.

Furthermore, we conducted the research in an inpatients psychiatric unit, so that the findings reported cannot be generalized to other clinical or outpatient samples likely affected by less severe disorders. Circumstances related to hospital admission may have rekindled memories of past experiences of violence, above all in early life, and may have influenced the patients' report.

\section{Conclusions}

This study offers a better understanding of how gender and age could affect violent behaviours. Moreover, our findings may increase the comprehension of the reason why some mental disorders, compared to others, are more associated with the risk of victimization (i.e., mood disorders) or perpetration of violence (i.e., personality disorders). These patterns could have pathophysiological or pathoplastic meaning addressing clinical and diagnostic trajectories and they could interact with other intervening risk factors.

\section{Abbreviations}

ANOVA: Analysis of variance; CATIE: Clinical Antipsychotic Trials of Intervention Effectiveness; IV: Interpersonal violence; KIVS: Karolinska Interpersonal Violence Scale; SMI: Severe mental illness;

SSOPD: Schizophrenia spectrum and other psychotic disorders

\section{Acknowledgements}

Not applicable.

\section{Authors' contributions}

DT made substantial contributions to study design, data acquisition, analyses, and interpretation of results; was involved in manuscript drafting and revision; gave final approval of the final version; and agreed to be accountable for all aspects of the work; AC: made substantial contributions to study design and interpretation of results; was involved in manuscript drafting and revision; AR: made substantial contributions to study design analyses, and interpretation of results; was involved in manuscript drafting and revision; PS: made substantial contributions to study design, analyses, and interpretation of results; was involved in manuscript drafting and revision; MM: made substantial contributions to study design, analyses, and interpretation of results; was involved in manuscript drafting and revision; FP: made substantial contributions to study design, analyses, and interpretation of results; was involved in manuscript drafting and revision; MC: made substantial contributions to data acquisition and was involved in manuscript drafting and revision; CC: made substantial contributions to data acquisition and was involved in manuscript drafting and revision; RR: made substantial contributions to data acquisition and was involved in manuscript drafting and revision. All authors have read and approved the manuscript.

\section{Funding}

This research did not receive any specific grant from funding agencies in the public, commercial, or not-for-profit sectors. 


\section{Availability of data and materials}

The datasets generated and analyzed during the current study are not publicly available as they contain individual-level data, but may be available from the corresponding author on reasonable request.

\section{Ethics approval and consent to participate}

All the procedures and the research project were approved by the local ethics committee (Internal Review Board of University of L'Aquila, approval number: 38204). Written informed consent was obtained from each participant prior to inclusion.

\section{Consent for publication}

Not applicable.

\section{Competing interests}

The authors declare that they have no competing interests.

\section{Author details}

'Department of Biotechnological and Applied Clinical Sciences (DISCAB), University of L'Aquila, L'Aquila, Italy. ${ }^{2}$ Department of Mental Health, ASL 1 Avezzano Sulmona L'Aquila, L'Aquila, Italy. ${ }^{3}$ Chair of Psychiatry, Department of Systems Medicine, University of Rome Tor Vergata, Rome, Italy.

\section{Received: 16 July 2019 Accepted: 12 April 2020}

\section{Published online: 01 May 2020}

\section{References}

1. Krug EG, Mercy JA, Dahlberg $L L, Z$ wi $A B$. The world report on violence and health. Lancet. 2002;360:1083-8. https://doi.org/10.1016/S01406736(02)11133-0

2. Sumner SA, Mercy JA, Dahlberg LL, Hillis SD, Klevens J, Houry D. Violence in the United States: status, challenges, and opportunities. JAMA. 2015;314: 478-88.

3. Rees S, Steel Z, Creamer M, Teesson M, Bryant R, McFarlane AC, et al. Onset of common mental disorders and suicidal behavior following women's first exposure to gender based violence: a retrospective, population-based study. BMC Psychiatry. 2014;14:312.

4. Rossi A, Talevi D. Interpersonal violence and mental illness. J Psychopathol. 2017;23:49-51

5. World Health Organization. Global Status Report on Violence Prevention 2014. Geneva; 2014

6. Rueve ME, Welton RS. Violence and mental illness. Psychiatry (Edgmont) 2008;5:34-48. https://doi.org/10.1136/bmj.289.6439.249-a.

7. Stuart H. Violence and mental illness : an overview. World Psychiatry. 2003;2: $121-4$.

8. Trevillion K, Oram S, Feder G, LM H. Experiences of domestic violence and mental disorders: a systematic review and meta-analysis. PLoS One. 2012;7: e51740.

9. Desmarais SL, Van Dorn RA, Johnson KL, Grimm KJ, Douglas KS, Swartz MS Community violence perpetration and victimization among adults with mental illnesses. Am J Public Health. 2014;104:2342-9.

10. Alnıak I, Erkıran M, Mutlu E. Substance use is a risk factor for violent behavior in male patients with bipolar disorder. J Affect Disord. 2016;193: 89-93.

11. Harford TC, Chen CM, Kerridge BT, Grant BF. Self- and other-directed forms of violence and their relationship with lifetime DSM-5 psychiatric disorders: results from the National Epidemiologic Survey on alcohol related conditions - III ( NESARC - III ). Psychiatry Res. 2016;2018(262):384-92. https://doi.org/10.1016/j.psychres.2017.09.012.

12. Latalova K, Kamaradova D, Prasko J. Violent victimization of adult patients with severe mental illness: a systematic review. Neuropsychiatr Dis Treat. 2014;10:1925-39.

13. Witt K, Van Dorn R, Fazel S. Risk factors for violence in psychosis : systematic review and meta-regression analysis of 110 studies. PLoS One. 2013;8: e55942.

14. Swanson J, Holzer C, Ganju V, Jono R. Violence and psychiatric disorder in the community : evidence from the epidemiologic catchment area surveys. Hosp Community Psychiatry. 1990:41:761-70.

15. Corrigan P, Watson A. Findings from the National Comorbidity Survey on the frequency of violent behavior in individuals with psychiatric disorders. Psychiatry Res. 2005;136:153-62.
16. Elbogen EB, Johnson SC. The intricate link between violence and mental disorder: results from the National Epidemiologic Survey on alcohol and related conditions. Arch Gen Psychiatry. 2009;66:152-61.

17. Fahlgren MK, Kleiman EM, Puhalla AA, McCloskey MS. Age and Gender Effects in Recent Violence Perpetration. J Interpers Violence. 2017. https:// doi.org/10.1177/0886260517709803.

18. Gillies D, Brien LO. Interpersonal violence and mental illness: a literature review. Contemp Nurse. 2006;21:277-86.

19. Moberg T, Stenbacka M, Jönsson EG, Nordström P, Åsberg M, Jokinen J. Risk factors for adult interpersonal violence in suicide attempters. BMC Psychiatry. 2014;14:195. https://doi.org/10.1186/1471-244X-14-195.

20. Jaen-Varas D, Mari J de J, Coutinho E da S, Andreoli SB, Quintana MI, de Mello MF, et al. A cross-sectional study to compare levels of psychiatric morbidity between young people and adults exposed to violence in a large urban center BMC Psychiatry. 2016;16:134. https://doi.org/10.1186/s12888016-0847-0

21. Hamberger LK. Men's and Women's use of intimate partner violence in clinical samples: toward a gender-sensitive analysis. Violence Vict. 2005;20: $131-51$

22. Robbins PC, Monahan J, Silver E. Mental Disorder, Violence, and Gender. Law Hum Behav. 2003;27:561-71.

23. Sorrentino R, Friedman SH, Hall R. Gender considerations in violence. Psychiatr Clin North Am. 2016;39:701-10. https://doi.org/10.1016/j.psc.2016. 07.002 .

24. de Waal MM, Dekker JJM, Kikkert MJ, Kleinhesselink MD, Goudriaan AE. Gender differences in characteristics of physical and sexual victimization in patients with dual diagnosis: a cross-sectional study. BMC Psychiatry. 2017; 17:270. https://doi.org/10.1186/s12888-017-1413-0 .

25. Meuleners L, Lee AH, Hendrie D. Effects of demographic variables on mental illness admission for victims of interpersonal violence. J Public Health (Bangkok). 2008;31:162-7.

26. Khalifeh H. Dean K. Gender and violence against people with severe mental illness. Int Rev Psychiatry. 2010;22:535-46.

27. Kmett JA, Eack SM. Characteristics of sexual abuse among individuals with serious mental illnesses. J Interpers Violence. 2018;33:2725-44. https://doi. org/10.1177/0886260516628811.

28. Temiz M, Beştepe E, Yildiz Ö, Küçükgöncü S, Yazici A, Çalikuşu C, et al. The effect of violence on the diagnoses and the course of illness among female psychiatric inpatients. Arch Neuropsychiatry. 2014;51:1-10.

29. Melton $\mathrm{HC}$, Sillito $\mathrm{CL}$. The role of gender in officially reported intimate partner abuse. J Interpers Violence. 2011;27:1090-111. https://doi.org/10. $1177 / 0886260511424498$.

30. American Psychiatric Association. Diagnostic and Statistical Manual of Mental Disorders: Diagnostic and Statistical Manual of Mental Disorders. 5th ed. Arlington: American Psychiatric Association; 2013

31. Jokinen J, Forslund K, Ahnemark E, Gustavsson J, Nordström P, Asberg M. Karolinska interpersonal violence scale predicts suicide in suicide attempters. J Clin Psychiatry. 2010;71:1025-32.

32. Sinai C, Hirvikoski T, Wiklander M, Nordström AL, Nordström P, Nilsonne $\AA$ et al. Exposure to interpersonal violence and risk of post-traumatic stress disorder among women with borderline personality disorder. Psychiatry Res. 2018;262:311-5. https://doi.org/10.1016/j.psychres.2018.01.047.

33. Jokinen J, Königsson J, Moberg T, Jönsson EG, Tiihonen J, Nordström P et al. Platelet monoamine oxidase activity and interpersonal violence in male suicide attempters. Psychiatry Res. 2018;260:173-6. https://doi.org/10. 1016/j.psychres.2017.11.057.

34. Bendix M, Uvnäs-moberg K, Petersson M, Kaldo V, Åsberg M, Jokinen J. Psychoneuroendocrinology insulin and glucagon in plasma and cerebrospinal fluid in suicide attempters and healthy controls. Psychoneuroendocrinology. 2017;81:1-7. https://doi.org/10.1016/j.psyneuen 2017.03.019.

35. Haglund A, Lindh ÅU, Lysell H, Renberg ES, Jokinen J, Waern M, et al. Interpersonal violence and the prediction of short-term risk of repeat suicide attempt. Sci Rep. 2016:6:36892. https://doi.org/10.1038/srep36892.

36. Stefansson J, Nordström P, Runeson B, Åsberg M, Jokinen J. Combining the suicide intent scale and the Karolinska interpersonal violence scale in suicide risk assessments. BMC Psychiatry. 2015;15:1-8. https://doi.org/10. 1186/s12888-015-0607-6.

37. Sinai C, Hirvikoski T, Nordström A, Nordström P, Nilsonne $\AA$, Wilczek A, et al. Thyroid hormones and adult interpersonal violence among women with borderline personality disorder. Psychiatry Res. 2015;227:253-7. 
38. Talevi D, Imburgia L, Luperini C, Zancla A, Collazzoni A, Rossi R, et al. Interpersonal violence: identification of associated features in a clinical sample. Child Abuse Negl. 2018;86:349-57. https://doi.org/10.1016/j.chiabu. 2018.08.017.

39. Rossi A, Talevi D, Collazzoni A, Parnanzone S, Stratta P, Rossi R. From basic human values to interpersonal violence: a mental illness sample. J Aggress Maltreat Trauma. 2020;29(3):259-271. https://doi.org/10.1080/10926771.201 9.1581865 .

40. Talevi D, Pacitti F, Costa M, Rossi A, Collazzoni A, Crescini C, Rossi R. Further Exploration of Personal and Social Functioning: The Role of Interpersonal Violence, Service Engagement, and Social Network. J Nerv Ment Dis. 2019; 207(10):832-7. https://doi.org/10.1097/NMD.0000000000001036.

41. Talevi D, Pacitti F, Parnanzone S, Crescini C, Lucaselli A, Costa M, et al. The Karolinska interpersonal violence scale, Italian version. J Psychopathol. 2019; 25:115-9.

42. Yu R, Geddes JR, Fazel S. Personality disorders, violence, and antisocial behavior : a systematic review and meta-regression analysis. J Personal Disord. 2012;26:775-92.

43. de Barros DM, de Pádua Serafim A. Association between personality disorder and violent behavior pattern. Forensic Sci Int. 2008;179:19-22.

44. Samuels J. Personality disorders: epidemiology and public health issues. Int Rev Psychiatry. 2011;23:223-33.

45. Howard R. Personality disorders and violence: what is the link? Borderline Personal Disord Emot Dysregulation. 2015;2:12. https://doi.org/10.1186/ s40479-015-0033-x.

46. Short V, Lennox C, Stevenson C, Senior J, Shaw J. Mental Illness, Personality Disorder and Violence: A Scoping Review. 2012. http://www.ohrn.nhs.uk/ OHRNResearch/Mlviolence.pdf.

47. Volavka J. Violence in schizophrenia and bipolar disorder. Psychiatr Danub. 2013;25:24-33.

48. Schick A, Cierpka M. Risk factors and prevention of aggressive behavior in children and adolescents. J Educ Res Online. 2016;8:90-109.

49. Steadman H, Monahan J, Pinals D, Vesselinov R, Robbins P. Gun violence and victimization of strangers by persons with a mental illness: data from the MacArthur violence risk assessment study. Psychiatr Serv. 2015;66:123841.

50. Swanson J, Swartz M, Van Dorn R, Elbogen E, Wagner H, Rosenheck R, et al. A National Study of violent behavior in persons with schizophrenia. Arch Gen Psychiatry. 2006;63:490-9.

\section{Publisher's Note}

Springer Nature remains neutral with regard to jurisdictional claims in published maps and institutional affiliations.

Ready to submit your research? Choose BMC and benefit from:

- fast, convenient online submission

- thorough peer review by experienced researchers in your field

- rapid publication on acceptance

- support for research data, including large and complex data types

- gold Open Access which fosters wider collaboration and increased citations

- maximum visibility for your research: over $100 \mathrm{M}$ website views per year

At $\mathrm{BMC}$, research is always in progress.

Learn more biomedcentral.com/submissions 